\title{
Remarks on Non Conventional Cardiac Electrical Activity Characterization from Implantable Cardioverter Defibrillator Signals
}

\author{
A Casaleggio ${ }^{1}, \mathrm{P} \mathrm{Rossi}^{2}, \mathrm{~T}_{\text {Guidotto }}{ }^{3}, \mathrm{~V}_{\text {Malavasi }}{ }^{4}$, \\ G Musso ${ }^{5}$, G Sartori $^{2}$ \\ ${ }^{1}$ Institute of Biophysics, National Research Council, Genova, Italy \\ ${ }^{2}$ Cardiology Division, San Martino Hospital, Genova, Italy \\ ${ }^{3}$ St Jude Medical Italia, Agrate Brianza, Milano, Italy \\ ${ }^{4}$ Cardiology Division, Modena Policlinic, Modena, Italy \\ ${ }^{5}$ Cardiology Service, Imperia Hospital, Imperia, Italy
}

\begin{abstract}
Implantable Cardioverter Defibrillators (ICDs) can store intracardiac electrograms (EGMs) with 2 modes of recording: bipolar (BIP) or far-field $(F F)$. Aims of this study are: (i) to propose a non conventional Cardiac Electrical Activity Characterization (CEAC); (ii) to investigate the mode of recording that better allows retrieving information from measured EGMs with CEAC.

We consider 293 EGMs during sinus rhythm (SR) or ventricular tachyarrhythmia (VT) from 40 patients implanted with ICDs. 196 were FF EGMs and 97 BIP EGMs. CEAC analysis of SR rhythms (FF vs. BIP) shows sensitivity $81.5 \%$ and specificity $93.6 \%$; with VT rhythms both were $100 \%$. CEAC analysis of FF signals (SR vs. $V T$ rhythms) give sensitivity 92.7 and specificity 79.7; with BIP EGMs they are $60 \%$ and $73.3 \%$, respectively.

We conclude that: (i) CEAC analysis requires to analyze FF or BIP signals separately; (ii) CEAC better discriminates SR from VT when far-field EGMs are used.
\end{abstract}

\section{Introduction}

New generation of implantable cardioverter defibrillators (ICDs) allow storage of growing amount of intracardiac electrograms (EGMs) immediately before the onset of the ventricular tachyarrhythmias (VT) and during their course with two different modes of recording: bipolar (BIP) and far-field (FF).. This opportunity has a clinical relevance for the understanding of mechanisms for malignant VT onset [1,2].

Bipolar EGM measures the electrical heart activity at the tip of the electrocatheter placed within the heart; the length of the dipole is about $1 \mathrm{~cm}$, allowing excellent monitoring of the local cardiac activity. Far-field EGM measures the electrical heart activity from the active device can and the distal coil of the electrocatheter; the length of the dipole is about $15 \mathrm{~cm}$ and it includes electrical cardiac activity of the whole heart. The two modes of recordings have different band-pass filters: BIP signals are filtered below $10-15 \mathrm{~Hz}$, while FF signals are filtered with a wider band-pass. In practice, while the bipolar EGMs allow more detailed analysis of the depolarization phase at the tip of the electrocatheter, the FF signals give an EGM more similar to the surface electrocardiogram.

This work proposes a cardiac electrical activity characterization (CEAC) based on power spectrum and correlation dimension estimation obtained from far-field and bipolar EGMs.

Next section resumes the data set and the quantitative parameters that we are using, then results will present the differences between CEAC obtained from EGM stored as FF or BIP (to explore if the two modes of recording may give differing conclusion about similar rhythms and thus EGMs obtained from the two modes should not be used together) and SR from VT rhythms (to verify the mode that allow better distinction between the two principal cardiac rhythms).

\section{Methods}

\section{Data set}

We consider 293 EGMs obtained from 40 patients (35 males and 5 females), average age 70 years, who underwent to ICD implantation as secondary prevention. The far-field group includes 196 EGMs from 25 patients whereas the bipolar group contains 97 EGMs from 25 patients. We separately analysed sinus rhythm (SR) and ventricular tachyarrhythmias (VT). To do it far-field and bipolar groups were divided into subgroups as follows: $\mathrm{VT}_{\mathrm{FF}}$ (far-field EGMs obtained during VT) which includes 90 EGMs from 23 patients, $\mathrm{SR}_{\mathrm{FF}}$ (far-field EGMs obtained during SR) which includes 106 EGMs from 25 patients, $\mathrm{VT}_{\mathrm{BIP}}$ (bipolar EGMs obtained during VT) which includes 48 EGMs from 16 patients and $\mathrm{SR}_{\mathrm{BIP}}$ 
(bipolar EGMs obtained during SR) which includes 49 EGMs from 21 patients.
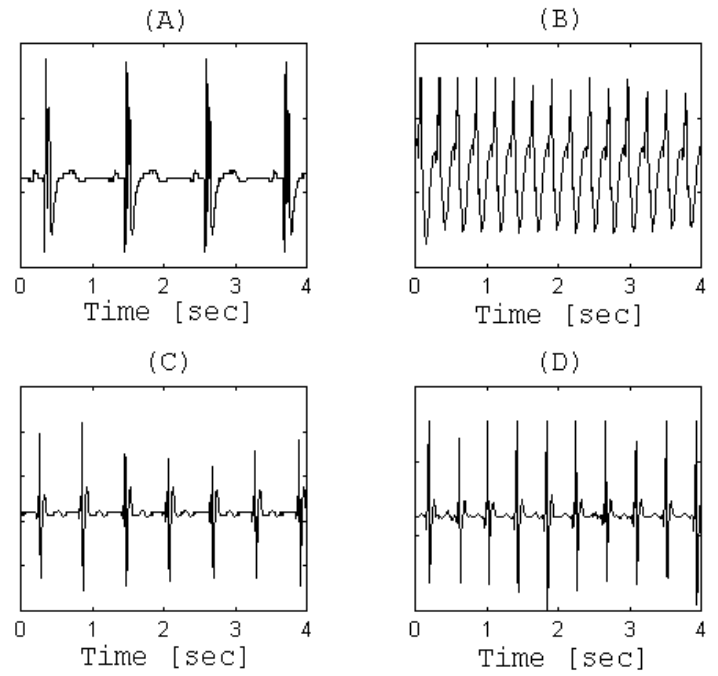

Figure 1: Example of FF and BIP EGMs obtained from ICDs. Panels A and B show far-field recordings obtained during SR and VT respectively. Similarly panels C and D present SR and VT recordings stored as bipolar.

\section{Methods of analyses}

The non-conventional cardiac electrical activity characterization (CEAC) is quantified by the frequency interval between the $10^{\text {th }}$ and $90^{\text {th }}$ percentile of the signal energy $\left(\mathrm{F}_{10-90}\right)$ and by correlation dimension $\left(\mathrm{D}_{2}\right)$, both estimated for all the EGMs. The power spectrum is computed using MATLAB routines, and we have fully tested software for $\mathrm{D}_{2}$ estimation whose details can be found in [3]. In order to quantify differences between CEAC obtained from far-field and bipolar electrograms, two statistic analysis are separately performed. The first of them, aimed to verify whether CEAC applied to BIP and FF EGMs is able to distinguish SR from VT rhythms, requires, for every mode of recording, to judge SR subgroup against VT subgroup and to verify that the two subgroups give differing results. The second statistic analysis has the purpose to verify the possibility that similar cardiac rhythms would be classified as pertaining to different classes depending on the mode of EGM recording (FF or BIP). In this case, for every cardiac rhythm, FF EGMs are compared with BIP ones.

The statistical analysis is based on sensitivity, specificity, positive and negative predictive values; in such a way we can respond to two questions: (i) does CEAC applied to FF or BIP EGMs distinguish between SR and VT cardiac rhythms? and (ii) does CEAC obtained from bipolar or far-field EGMs during similar cardiac rhythms give similar results?

\section{Results}

\section{Qualitative analysis}

Qualitative results obtained from EGMs of Fig. 1 are shown in the next two Figures. In particular Fig. 2 shows the power spectra of the 4 EGMs of Fig.1, while Fig. 3 shows their correlation integrals. In particular the Fig. 3 shows the set of functions $f(m, r)$ (defined as derivative of the logarithm of the correlation integral versus the logarithm of the correlation length) with their corresponding correlation dimensions obtained from the plateau of $f(m, r)$ (see [3] and reference therein for details).
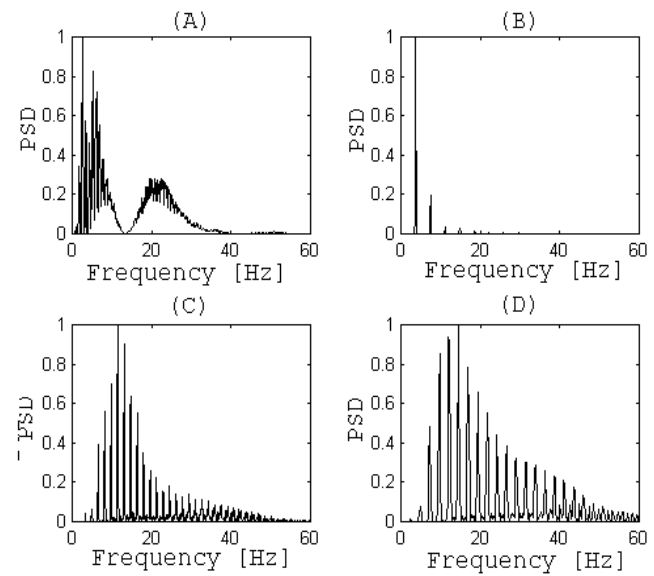

Figure 2: Power spectra from the same EGMs as in Figure 1. Panels A and B are related to far-field recordings of SR and VT, while panels $\mathrm{C}$ and $\mathrm{D}$ regard bipolar recordings during SR and VT, respectively.


Figure 3: Examples of $f(m, r)$ estimated from the same EGMs as in Figure 1. Panels A and B are related to far-field recordings during SR and VT, while panels $\mathrm{C}$ and $\mathrm{D}$ regard bipolar recordings during SR and VT. The computation of the set of functions $f(m, r)$ are fully described in [3]. 


\section{Quantitative analysis}

Quantitative description of CEAC differences between the 4 subgroups is resumed in Table 1 and Table 2. In particular Table 1 investigates the possibility to distinguish cardiac rhythms (SR from VT) using CEAC applied to FF or BIP EGMs. Table 2 explore the influence of the choice of the mode of recording in the CEAC classification of SR or VT signals. The tables include the information of the number of EGM contained in every subgroup, while the differences are presented in terms of ability to separate EGMs pertaining to different subgroups in terms of sensitivity, specificity, positive and negative predictive values.

Table 1: Differences between CEAC of SR and VT EGMs for bipolar and far-field modes of recording.

\begin{tabular}{lllllll}
\hline Mode & \#SR & \#VT & Sens. & Spec. & PPV & NPV \\
\hline FF & 106 & 90 & $92.7 \%$ & $79.7 \%$ & $77.3 \%$ & $93.7 \%$ \\
\hline BIP & 49 & 48 & $60 \%$ & $73.3 \%$ & $42.9 \%$ & $84.6 \%$ \\
\hline
\end{tabular}

Table 2: Differences between CEAC of SR and VT EGMs as a consequence of the choice of the mode of recording.

\begin{tabular}{lllllll}
\hline Rhythm & \#FF & \#Bip & Sens. & Spec. & PPV & NPV \\
\hline SR & 106 & 49 & $81.5 \%$ & $93.6 \%$ & $92.1 \%$ & $84.6 \%$ \\
\hline VT & 90 & 48 & $100 \%$ & $100 \%$ & $100 \%$ & $100 \%$ \\
\hline
\end{tabular}

\section{Discussion and conclusions}

There is a common believe that not all information contained in intracardiac electrograms are extracted from them and made easily understandable to the physicians. This is being an open field of research and great effort is spending to investigate methods to improve the recognition of cardiac patients with higher risk of developing malignant tachyarrhythmias $[4,5]$ on the basis of information extracted from the EGMs.

As a non-conventional type of analysis, different from the various methods that have already been proposed, we chose two simple and straight-forward types of analysis for our cardiac electrical activity characterization (CEAC). The first of them is aimed to investigate the range of frequencies containing the large majority of signal energy; it is quantified as the range between the frequency of $10^{\text {th }}$ and $90^{\text {th }}$ percentiles of the signal energy, and it is called $f_{10-90}$. The second approach explore the variability of the whole beat signals; it is quantified by the correlation dimension estimation, and it is called $D_{2}$.

In this explorative paper we do not address a clinical question, and it is not our purpose to investigate methods for risk stratification of patients with cardiac illnesses.
On the contrary we carry out a preliminary study aimed to explore the mode of EGM recording that appears as the most promising when the analysis is aimed to classify EGMs with CEAC. Essentially only two modes of EGM recording are available: bipolar and far-field. Purpose of this paper is to compare CEAC obtained from far-field against bipolar EGMs to investigate the mode that allows better performances of the CEAC.

Preliminarily it is known that bipolar recordings give detailed information about the electrical activity, locally within the heart; it is also known that they are focused on the monitoring of the depolarization phase, indeed only very seldom they reveal the T-wave pattern; finally the filer band-pass that devices uses for bipolar EGMs has a narrow band-pass in order to eliminate spurious electrical activities not directly due to the electrical heart activity. On the contrary far-field EGMs monitor a larger dipole that includes depolarization and repolarization (the Twave is often clearly visible in far-field signals), and they are filtered with a wider band-pass.

Some of the previous consideration are qualitatively supportable by looking at the Figures. In Fig. 1 we can note that the $\mathrm{T}$-wave is visible in panel $\mathrm{A}$, where a $\mathrm{FF}$ EGM during SR is shown, but not in panel $\mathrm{C}$ that present an example of BIP EGM during SR. In Fig. 2 we can qualitatively observe two things: (i) looking at the difference between panels A (during SR) and B (during VT) we note that the power spectrum of a FF EGM strongly changes with the cardiac rhythm; (ii) looking at the patterns of panels $\mathrm{C}$ and $\mathrm{D}$ we observe that they remind the power spectrum of a train of impulses, for this reason we do not get a large change in the pattern when the cardiac rhythm changes: indeed we simply get a slight change in the distance between following impulses, and we can say that it is due to the different beat rate and it can be more easily accounted by the quantification of the cardiac cycle. By the way, this result is consistent with the algorithm for data compression previously presented [6] where bipolar signals were compressed simply storing a short interval around the $\mathrm{R}$ peak along with the $\mathrm{RR}$ intervals, while all the rest of the signal is ignored.

In Fig. 3 it should be noted how BIP EGMs do not evidence any plateau in the $f(m, r)$ set of functions, while FF EGMs do. This means that in the case of bipolar EGMs the number of missing values because of an undetermined in $\mathrm{D}_{2}$ estimation is very high. Another observation is related to the slightly higher $\mathrm{D}_{2}$ that we observe from the analysis of VT rhythm in FF EGMs. Although the variation is small, it is significant as it will be shown by the quantitative analysis.

Next comments regard quantitative analyses. Table 1 indicates the type of classification between SR and VT rhythms that can be achieved with FF and BIP signals. Although values of sensitivity and specificity as $92.7 \%$ 
and $79.7 \%$ respectively can not be considered as excellent results, these results indicate that CEAC applied to FF signals distinguish better the two cardiac rhythms than when BIP EGMs are considered (for which we obtain sensitivity of $60 \%$ and specificity of $73.3 \%$ ). This indicates that FF mode of recording allows better performances of the CEAC based on $f_{10-90}$ and $D_{2}$ parameters. Table 2 explore the differences between FF and BIP for similar cardiac rhythm. Ideally results should be similar, but this is not the case: indeed we get very well separated classes in the case of VT rhythms, and a quite high sensitivity and specificity values also for the SR rhythms. That means that the choice of the mode of recording influence significantly the outcomes of the analysis with our CEAC.

Final comments regard drawbacks of this work: the major pitfall is the lack of simultaneous BIP and FF recordings. We think we overwhelm this pitfall by presenting a significant statistics: about 300 intracardiac electrograms quite well distributed among the 4 subgroups (the smallest subgroup count 48 EGMs). Another drawback is related to the statistic analysis: we considered all the EGMs as independent each other. This is not true: indeed we have many records from some patients and less from others. So a complementary statistical analysis should be carried on averaged CEAC results obtained from every patient. This analysis is subject of a study that we are carrying on. Preliminary results are consistent with those presented in this paper, although the final paper has not been published yet.

In conclusion this paper investigates the two principal modes for intracardiac electrograms recording and storage that are commonly implemented in ICD: bipolar and farfield.

The cardiac electrical activity characterization (CEAC) is defined on the basis of frequency range of EGMs energy and on correlation dimension estimation.

Results indicate that bipolar and far-filed EGMs give significantly different CEAC values when applied to EGM obtained from similar cardiac rhythms (SR and VT), thus BIP and FF signals should be analyzed separately. Secondly CEAC distinguishes EGM stored during SR from EGM recorded during VT rhythms with higher sensitivity and specificity when the mode of recording is far-field.

\section{References}

[1] Sarter BH, Callans DJ, Gottlieb GD, Schwartzman DS, Marchlinski FE. Implantable defibrillator diagnostic storage capabilities: evolution, current status, and future utilization. PACE 1998;21:12871298

[2] Auricchio A, Hartung W, Geller C, Klein H. Clinical relevance of stored electrograms for implantable cardioverter-defibrillator (ICD) troubleshooting and understanding of mechanisms for ventricular tachyarrhythmias. Am J Cardiol. 1996; 78:33-41.

[3] Corana A, Bortolan G, Casaleggio A. "Most probable dimension value and Most flat interval methods for automatic estimation of dimension from time series" Chaos Solitons Fractals 20: 779-790, 2004.

[4] Pedretti RF, Sarzi Braga S. "Non-invasive sudden death risk stratification." Ital Heart J. 20056 180189. Review.

[5] Hohnloser SH, Klingenheben T, Li YG, Zabel M, Peetermans J, Cohen RJ. T wave alternans as a predictor of recurrent ventricular tachyarrhythmias in ICD recipients: prospective comparison with conventional risk markers. J Cardiovasc Electrophysiol. 1998 9:1258-1268

[6] Rossi P, Casaleggio A, Chiappalone M, Morando M, Corrucci G, Reggiani M, Sartori G, Chierchia S. "Computationally inexpensive methods for intracardiac atrial bipolar electrogram compression" EuroPace 4:295-302, 2002.

Address for correspondence
Aldo Casaleggio
IBF - CNR, Via De Marini, 6
16149, Genova, Italy.
E-mail address: casaleggio@ge.ibf.cnr.it 